08,03

\title{
Влияние неравновесных корреляций на эффективные сопротивления между центрами в теории прыжковой проводимости
}

\author{
() А.В. Шумилин, Я.М. Бельтюков \\ Физико-технический институт им. А.Ф. Иофрфе РАН, \\ Санкт-Петербург, Россия \\ E-mail: avshumilin@mail.ioffe.ru
}

Поступила в Редакцию 10 июня 2019 г.

В окончательной редакции 10 июня 2019 г.

Принята к публикации 10 июня 2019 г.

\begin{abstract}
Показано, что неравновесные корреляции в прыжковой проводимости могут быть учтены с помощью обобщения сетки сопротивлений Миллера-Абрахамса. Сопротивления резисторов между центрами в такой сетке связаны их окружением. Кроме того, возникают нелокальные элементы, создающие напряжение на одном резисторе, пропорциональное току через другой. Эти элементы нужны для того, чтобы сделать приближения, использованные при построении сетки, контролируемыми. Наиболее сильное изменение сопротивления возникает в резисторах с сопротивлением меныше критического сопротивления теории протекания.
\end{abstract}

Ключевые слова: прыжковая проводимость, корреляции чисел заполнения, сетка сопротивлений Миллера-Абрахамса.

DOI: 10.21883/FTT.2019.11.48416.509

\section{1. Введение}

Наиболее известным инструментом для теоретического изучения прыжковой проводимости на сегодняшний день является сетка сопротивлений Миллера-Абрахамса [1]. Эта сетка является начальной точкой для теории протекания, с помощью которой могут быть выведены температурные зависимости проводимости в режимах Мотта и Эфроса-Шкловского. Тем не менее, сетка сопротивлений Миллера-Абрахамса построена на приближении среднего поля, которое является неконтролируемым в случае, когда в прыжковой проводимости принимают участия состояния по разные стороны от уровня Ферми [2].

В рамках приближения среднего поля предполагается, что среднее произведение чисел заполнения $\overline{n_{i} n_{j}}$ на двух центрах $i$ и $j$ равняется произведению средних чисел заполнения $\bar{n}_{i} \bar{n}_{j}$. При этом не учитываются корреляции чисел заполнения, например ковариация $\overline{n_{i} n_{j}}-\bar{n}_{i} \bar{n}_{j}$. Такие корреляции могут возникать либо из-за кулоновского отталкивания носителей на центрах $i$ и $j$ либо изза выведения системы из равновесия. Без кулоновского взаимодействия и в равновесии $\overline{n_{i} n_{j}}-\bar{n}_{i} \bar{n}_{j}=0$. В настоящей работе мы не рассматриваем дальнодействующее кулоновское взаимодействие, однако предполагается, что отталкивание носителей заряда не позволяет двум носителям находиться на одном центре. Соответственно, предметом исследования является вторая причина возникновения корреляций: отклонение состояния системы от равновесного, например, из-за приложенного электрического поля.

Известно, что даже в слабом электрическом поле, в режиме линейного отклика, неравновесные корреляции могут быть важны для теории транспорта в некоторых прыжковых системах. Так, приближение среднего поля неверно оценивает энергию активации при транспорте в цепочке из двух видов центров [3]. В полупроводниках в режиме закона Мотта проводимость, рассчитанная с помощью сетки сопротивлений Миллера-Абрахамса, отличается от реальной на величину порядка единицы [4]. Таким образом, теория среднего поля описывает проводимость только с экспоненциальной точностью. Кроме того, существуют явления, напрямую связанные с неравновесными корреляциями. Так, магнетосопротивление, наблюдаемое во многих органических полупроводниках и получившее название „органическое магнетосопротивление“ или „ОМAR“ [5], определяется влиянием магнитного поля на скорость релаксации неравновесных спиновых корреляций. Это явление не может быть описано в рамках среднего поля, поскольку в этой модели спиновые корреляции не влияют на проводимость [6]. Учет спиновых корреляций даже в очень упрощенной модели позволяет описать связь спиновых корреляций и транспорта и объяснить эффект OMAR [7].

На сегодняшний день существует не очень много теоретических приемов, позволяющих строить теорию прыжковой проводимости с учетом неравновесных корреляций. Наиболее известный такой прием - расчет методом Монте-Карло. Этот метод позволяет численно рассчитать проводимость с учетом всех корреляций, однако плохо подходит для построения аналитической теории. Практически единственной альтернативой этому методу до недавнего времени было рассмотрение уравнений в пространстве многочастичных состояний. Такие уравнения могут быть сведены к сетке сопротивлений, содержащей $2^{N}$ узлов, где $N$ - число центров 
в системе [8]. Также существует возможность учесть корреляции в близких парах центров как поправки к сопротивлениям Миллера-Абрахамса [9].

Недавно авторами этой статьи был предложен новый метод теоретического исследования прыжковой проводимости, основанный на системе корреляционных кинетических уравнений. Вместо $2^{N}$ состояний системы такая система уравнений оперирует с числами заполнения и всеми возможными их корреляциями. Так как корреляции между удаленными центрами, как правило, малы, это позволяет обрезать систему уравнений и рассматривать число неизвестных, существенно меньшее чем $2^{N}[10]$. В режиме линейного отклика системе кинетических уравнений для корреляций может быть поставлена в соответствие эквивалентная электрическая схема, обобщающая сетку сопротивлений Миллера-Абрахамса. Важным недостатком эквивалентной схемы [10] является то, что число узлов в ней все же велико (много больше $N$ ), хоть и много меньше, чем $2^{N}$. Из-за этого теряется наглядность представления Миллера-Абрахамса. В настоящей работе мы показываем, что, используя подход кинетических уравнений, можно вернуться к эквивалентной схеме, узлы которой соответствуют прыжковым центрам. Однако для того, чтобы сохранить при этом строгость теории, необходимо внести в нее нелокальные элементы, связывающие напряжение на одной паре узлов с током между узлами другой пары. Тем не менее, наши расчеты показывают, что даже без учета этих нелокальных элементов построенная таким образом сетка сопротивлений достаточно хорошо описывает токи, текущие в реальной системе.

\section{2. Система корреляционных кинетических уравнений}

В этом разделе мы кратко изложим результаты [10], необходимые для понимания настоящей работы.

В рамках теории линейного отклика корреляционные кинетические уравнения могут быть сведены к эквивалентной электрической схеме. Узлы этой схемы соответствуют любым множествам прыжковых центров. Например, узел $I=\{i, j, k\}$ соответствует тройке центров $i-j-k$. Множества из одного элемента соответствуют одиночным прыжковым центрам (узлам сетки сопротивлений Миллера-Абрахамса).

Потенциал $\bar{u}_{I}$ на узле $I$ может быть связан с физически-измеримой величиной $\overline{\delta n}_{I}$

$$
\begin{gathered}
\overline{\delta n}_{I}=\prod_{i \in I} n_{i}^{(0)}\left(1-n_{i}^{(0)}\right) \bar{u}_{I}, \\
\overline{\delta n}_{I}=\overline{\prod_{i \in I} n_{i}-n_{i}^{(0)}} .
\end{gathered}
$$

Здесь $n_{i}^{(0)}-$ равновесное значение среднего числа заполнения центра $i$. В рамках теории линейного отклика
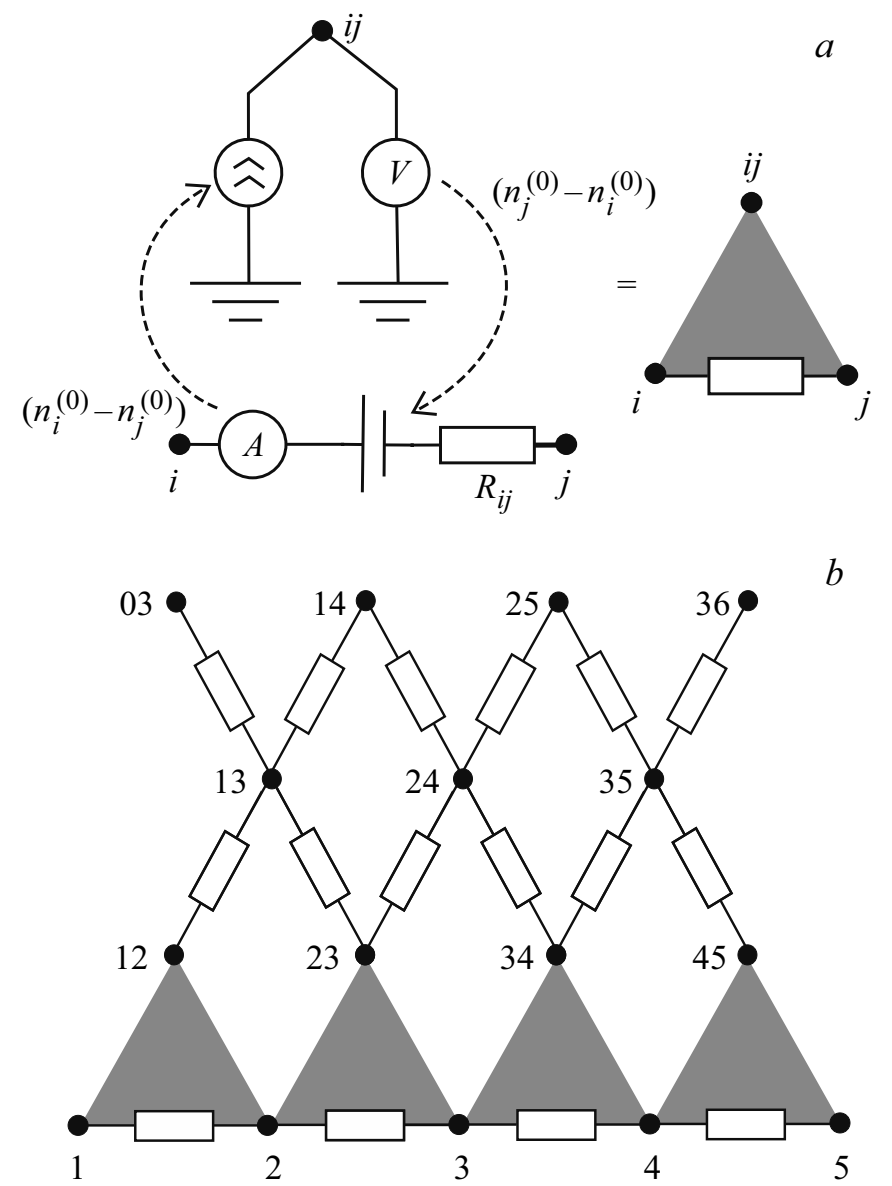

Рис. 1. (a) связь между уровнями эквивалентной схемы [10]. (b) пример эквивалентной схемы, содержащей пять узлов и парные корреляции между ними.

$\overline{\delta n}_{I}$ равняется ковариации чисел заполнения центров в множестве $I$.

Центры в эквивалентной схеме разделены на уровни. Каждый уровень соответствует определенному количеству $m_{I}$ элементов в множестве $I$. В рамках одного уровня центры соединены сопротивлениями. В случае если множества $I_{1}=I^{\prime} \cup i$ и $I_{2}=I^{\prime} \cup j$ отличаются одним элементом, сопротивление резистора $R_{I_{1}, I_{2}}$ можно записать как

$$
R_{I_{1}, I_{2}}=R_{i j}^{(M A)}\left(\prod_{i \in I^{\prime}} n_{i}^{(0)}\left(1-n_{i}^{(0)}\right)\right)^{-1} .
$$

Здесь $R_{i j}^{(M A)}$ - сопротивление резистора МиллераАбрахамса между центрами $i$ и $j$. Для множеств $I_{1}$ и $I_{2}$, отличающихся более чем одним центром, резистора между соответствующими узлами нет. Первый уровень эквивалентной схемы воспроизводит сетку сопротивлений Миллера-Абрахамса.

Связь между уровнями схемы изображена на рис. $1, a$. За счет каждой пары узлов $i-j$ принадлежащих множеству $I$, в узел, соответствующий этому множеству, 
втекает ток

$$
\widetilde{J}_{I}^{(i j)}=\left(n_{i}^{(0)}-n_{j}^{(0)}\right) J_{I \backslash i, I \backslash j}
$$

Здесь $J_{I \backslash i, I \backslash j}$ - ток, который течет уровнем ниже между узлами, соответствующими множествам $I \backslash i$ и $I \backslash j$. Обозначение $I \backslash i$ означает удаление из множества $I$ центра $i$ (вычитание множеств).

При этом потенциал $\bar{u}_{I}$ создает дополнительное напряжение между узлами $I \backslash i$ и $I \backslash j$, равное $\left(n_{j}^{(0)}-n_{i}^{(0)}\right) \bar{u}_{I}$. На рис. $1, b$ изображена часть эквивалентной схемы, соответствующая цепочке из пяти центров и парным корреляциям между ними.

Полное число узлов эквивалентной схемы соответствует полному числу подмножеств множества узлов в системе и равно $2^{N}$, где $N-$ число узлов. Тем не менее, в большинстве случаев для физического описания системы достаточно учитывать ограниченный набор корреляций не слишком высокого порядка и между центрами, лежащими достаточно близко друг к другу. В этом случае можно пренебречь какой-то частью корреляций. С точки зрения эквивалентной схемы необходимо считать потенциал $\bar{u}_{I}$ на соответствующих узлах равным нулю, то есть, заземлить эти узлы.

Известно, что уравнения Кирхгофа в эквивалентной схеме могут быть сведены к задаче о минимизации функционала $F=F_{0}+F_{S}$. Здесь

$$
F_{0}=\sum_{\substack{I, i, k \\ i<k}} \frac{\left[\bar{u}_{I \cup k}-\bar{u}_{I \cup i}+\left(n_{k}^{(0)}-n_{i}^{(0)}\right) \bar{u}_{I \cup i \cup k}\right]^{2}}{2 R_{I \cup i, I \cup k}}
$$

связан с потенциалами $\bar{u}_{I}$ на узлах. $F_{S}-$ функционал, соответствующий источнику, выводящему систему из равновесия. В частности, в работе [10] такие источники определялись различием электрических потенциалов на парах центров $\varphi_{i j}=\mathbf{r}_{i j} \mathbf{E}$. Для настоящей работы важен другой вид функционала источника, связанный с внешними токами, втекающими в узлы схемы. Если в некоторые узлы эквивалентной схемы втекают внешние токи $J_{I}^{\mathrm{ext}}$, функционал $F_{S}$ может быть записан в виде

$$
F_{S}=\sum_{I} \bar{u}_{I} J_{I}^{\mathrm{ext}}
$$

Слагаемое $\left(n_{k}^{(0)}-n_{i}^{(0)}\right) \bar{u}_{I \cup i \cup k}$ в (5) определяет взаимосвязь между уровнями эквивалентной схемы, которая изображена на рис. 1.

Выражение (5) является квадратичной формой, поэтому $F$ можно записать в виде

$$
F=\frac{1}{2} \sum_{I, K} M_{I, K} \bar{u}_{I} \bar{u}_{K}+\sum_{I} \bar{u}_{I} J_{I}^{\mathrm{ext}},
$$

где $M$ - квадратная симметричная положительно определенная матрица. Задача о минимизации $F$ и нахождения потенциалов $\bar{u}_{I}$ сводится к решению системы линейных уравнений

$$
\sum_{K} M_{I, K} \bar{u}_{K}=-J_{I}^{\mathrm{ext}}
$$

Заметим, что обычная схема из резисторов также описывается системой линейных уравнений с симметричной положительно определенной матрицей.

\section{3. Описание системы с помощью сетки сопротивлений и транс-сопротивлений}

Описанная выше эквивалентная схема достаточно удобна для численного моделирования с помощью уравнений Кирхгофа. Тем не менее, она содержит большое количество узлов, связанных с корреляциями. Как правило, число таких узлов оказывается значительно большим, чем количество узлов в сетке сопротивлений Миллера-Абрахамса. Это делает эквивалентную схему [10] достаточно сложной для анализа.

Существует возможность построить описание, используя только узлы, соответствующие прыжковым центрам. При этом сопротивления между парами центров должны быть пересчитаны с учетом окружения этих пар. Кроме того в системе возникают транс-сопротивления, связывающие токи через одну пару центров с напряжением на другой паре центров.

Для того чтобы вывести новую схему воспользуемся линейностью уравнений Кирхгофа. С точки зрения эквивалентной схемы [10] возникновение корреляций связано с токами $\widetilde{J}^{(i j)}$, втекающими на второй уровень. Так как уравнения линейны, любой корреляционный потенциал $\bar{u}_{I}$ можно разложить на слагаемые, связанные с различными парами центров $(i j)$ :

$$
\bar{u}_{I}=\sum_{i j} \eta_{I}^{(i j)} \widetilde{J}^{(i j)}
$$

Для того чтобы рассчитать величины $\eta_{I}^{(i j)}$ с заданными индексами $(i j)$, необходимо рассмотреть „обрезанную“ схему, изображенную на рис. 2. В этой схеме первый уровень (соответствующий прыжковым центрам) заменен на единичный ток, втекающий в пару узлов $i j$. Вся остальная схема [10] (включая связи между верхними

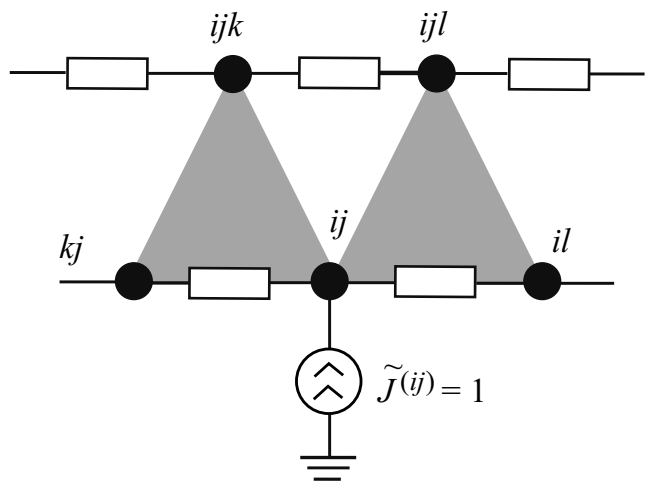

Рис. 2. „Обрезанная“ эквивалентная схема, позволяющая рассчитывать транс-сопротивленния. 


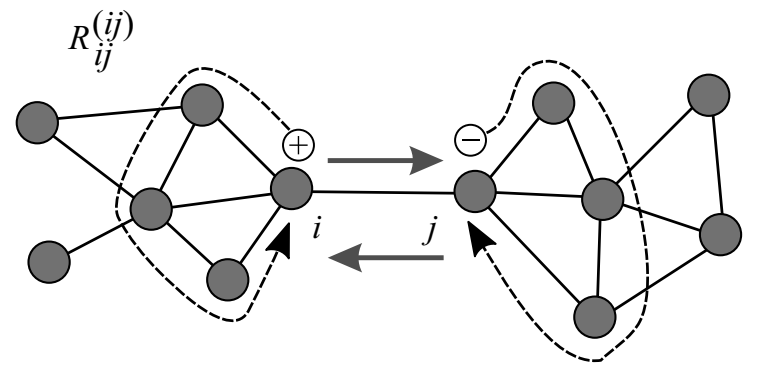

$a$

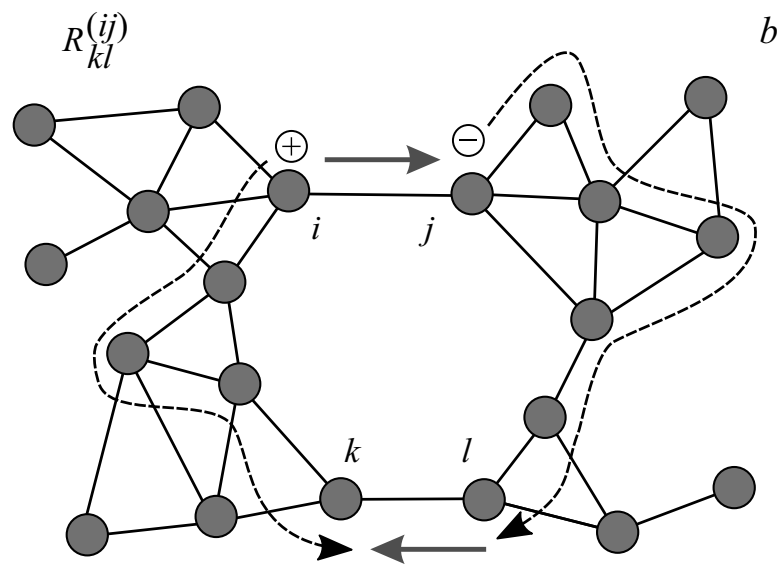

Рис. 3. Качественный мезназим возникновения корреляционных поправок к сопротивлениям Миллера-Абрахамса $(a)$ и транс-сопротивлений $(b)$.

уровнями) остается без изменений, поэтому она может быть описана с помощью системы уравнений (8). При этом в системе уравнений нужно положить отличным от нуля только один втекающий ток $J_{(i j)}^{\mathrm{ext}}=\widetilde{J}^{(i j)}$. Потенциалы, найденные при решении этой задачи, мы обозначим $\eta_{I}^{(i j)}$.

С точки зрения реальных токов между прыжковыми центрами, эффект корреляций сводится к появлению дополнительных напряжений. С учетом разложения (9) эти напряжения можно свести к набору транссопротивлений $R_{k l}^{(i j)}$

$$
\begin{gathered}
\delta U_{k l}=\sum_{i j} R_{k l}^{(i j)} J_{i j}, \\
R_{k l}^{(i j)}=\left(n_{k}^{(0)}-n_{l}^{(0)}\right)\left(n_{i}^{(0)}-n_{j}^{(0)}\right) \eta_{(k l)}^{(i j)} .
\end{gathered}
$$

При одинаковых верхних и нижних индексах $R_{i j}^{(i j)}$ соответствуют поправкам к сопротивлению, которые должны добавляться к обычным сопротивлениям резисторов Миллера-Абрахамса. При различных индексах $R_{k l}^{(i j)}$ соответствуют транс-сопротивлениям. Заметим, что транс-сопротивления обладают симметрией $R_{k l}^{(i j)}=R_{i j}^{(k l)}$, поскольку коэффициенты $\eta_{(k l)}^{(i j)}$ определялись путем решения системы линейных уравнений с симметричной матрицей.

Качественная картина, описывающая возникновение поправок к сопротивлению и транс-сопротивлений, показана на рис. 3. В теории среднего поля проводимость резистора $i-j$ пропорциональна среднему количеству электронов, прыгающих в этой паре в равновесии (в любом направлении) [2]. В результате прыжка $i \rightarrow j$ одновременно образуется электрон на центре $j$ и свободное место (дырка) на центре $i$. Их одновременное появление описывается корреляцией $\overline{\delta n}_{(i, j)}$. В том случае, если эти электрон и дырка (возможно, после каких-то перемещений между центрами) рекомбинировали друг с другом на той же паре узлов $i-j$, прыжок $i \rightarrow j$ не приводит к реальному перемещению заряда в системе (см. рис. 3,a). Подобные события следует исключить во время расчета проводимости резистора $i-j$. Это исключение соответствует корреляционной поправке к сопротивлению резистора $R_{i j}^{(i j)}$. Транс-сопротивления $R_{k l}^{(i j)}$ соответствуют следующим физическим процессам. Электрон-дырочная пара рождается на паре центров $i j$, а рекомбинирует на другой паре центров $k l$ (см. рис. $3, b$ ).

В случае если $i j-$ один из немногих резисторов, разделяющих две относительно хорошо проводящих области (например критический резистор теории протекания [2]), вероятность того, что электрон-дырочная пара, появившаяся на центрах $i j$ рекомбинирует на другой паре центров, может оказаться малой. В этом случае можно пренебречь транс-сопротивлениями $R_{k l}^{(i j)}$ и сохранить в теоретическом описании только поправки $R_{i j}^{(i j)}$. В общем случае, однако, такое приближение является неконтролируемым.

\section{4. Изменение сопротивлений резисторов сетки Миллера-Абрахамса за счет корреляций}

В рамках этой работы мы сравниваем подход с использованием транс-сопротивлений с прямым решением корреляционных кинетических уравнений. Мы сравниваем подходы путем численных экспериментов на образцах со статистикой центров соответствующей режиму закона Мотта. Образцы содержат 100 центров, случайно расположенных в квадрате $10 \times 10$. Таким образом, концентрация центров определяет единицу измерения длины. Интегралы перекрытия между центрами $t_{i j}$ экспоненциально спадают с расстоянием как $t_{i j}=t_{0} \exp \left(-r_{i j} / a\right)$. Здесь $r_{i j}$ - расстояние между центрами $i$ и $j, a=0.2-$ радиус локализации, а $t_{0}$ - некоторая константа. Энергии центров случайно разбросаны в интервале $(-1,1)$, то есть единицы измерения энергии соответствуют разбросу энергий центров. Такая статистика интегралов перекрытия и энергии соответствуют закону Мотта, однако размер наших систем недостаточен, чтобы считать их макроскопическими.

На рис. 4 приведена зависимость отношения проводимости с учетом корреляции к проводимости, рассчитанной в приближении Миллера-Абрахамса. Результаты усреднены по пятидесяти численным образцам. Сплош- 


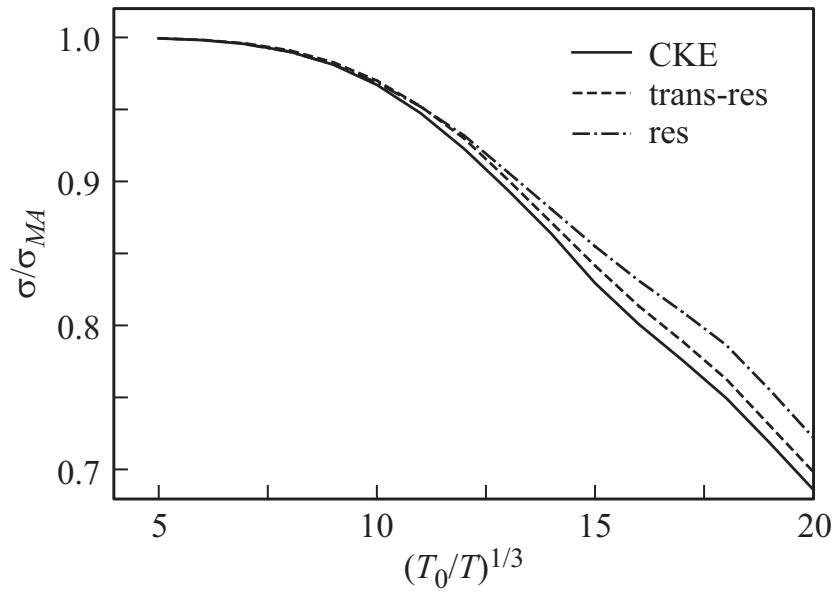

Рис. 4. Отношение проводимости, рассчитанной с учетом корреляций, к проводимости, рассчитанной по модели Миллера-Абрахамса. Корреляции учтены тремя различными способами: с помощью решения системы кинетических уравнений (CKE), с помощью транс-сопротивлений (trans-res) и с помощью поправок к сопротивлениям резисторов (res).

ной линией обозначен результат, полученный с помощью численного решения корреляционных уравнений. При этом учитывались все парные корреляции между центрами на расстоянии до $2 r_{c}$, где $r_{c}=a \xi_{c} / 2-$ критическая длина прыжка в теории протекания. Пунктирной линией показан расчет с помощью транс-сопротивлений. При этом при расчете транс-сопротивлений также учитывались парные корреляции при расстоянии между центрами не больше $2 r_{c}$, но только между центрами, которые расположены не дальше чем на расстоянии $3 r_{c}$ от пары центров $i j$, порождающей корреляцию. Таким образом, полное число учтенных корреляций оказывается меньше, из-за этого пунктирная линия на рис. 1 отличается от сплошной.

Штрих-пунктирная линия на рис. 1 соответствует расчету, в котором не учитываются транс-сопротивления $R_{k l}^{(i j)}$ если пара $k l$ отличается от пары $i j$. Таким образом, эквивалентная схема, соответствующая штрихпунктирной линии на рис. 4 , представляет собой обычную сетку сопротивлений между центрами, где величины сопротивлений рассчитаны с учетом корреляций.

Пренебрежение транс-сопротивлениями еще немного увеличивает рассчитанное значение проводимости, тем не менее, оно все еще оказывается близко к значению, полученному с помощью системы кинетических уравнений. Это дает возможность поставить вопрос, как меняются значения сопротивлений между центрами при учете неравновесных корреляций.

Такие результаты приведены на рисунках 5 и 6. Каждая точка на графике 5 соответствует одному резистору. По горизонтальной оси в логарифмическом масштабе отложено отношение величины сопротивления резистора к сопротивлению критического резистора теории протекания. По вертикальной - относительное изменение сопротивления резистора по отношению к сопротивлению Миллера-Абрахамса. Расчет показывает, что при учете корреляций величина всех сопротивлений возрастает. Тем не менее, эффект очень сильно различается для различных резисторов. Для одних резисторов такое увеличение незначительно, для других сопротивление может увеличиться на порядки. Как видно из рис. 5, сильнее всего (в среднем) этот эффект проявляется для резисторов с маленьким сопротивлением. Изменение сопротивления резисторов с сопротивлением, большим по сравнению с критическим, как правило, мало. Тем не менее, существуют резисторы, с сопротивленим, сопоставимым по величине с критическим, которое увеличиваются из-за корреляций в десятки раз.

На рис. 6 изображена взаимосвязь изменения сопротивления резистора и протекающего через него тока. Как и на рис. 5, каждая точка соответствует одному резистору. Ток измерен в единицах $J_{C}$, соответствующих

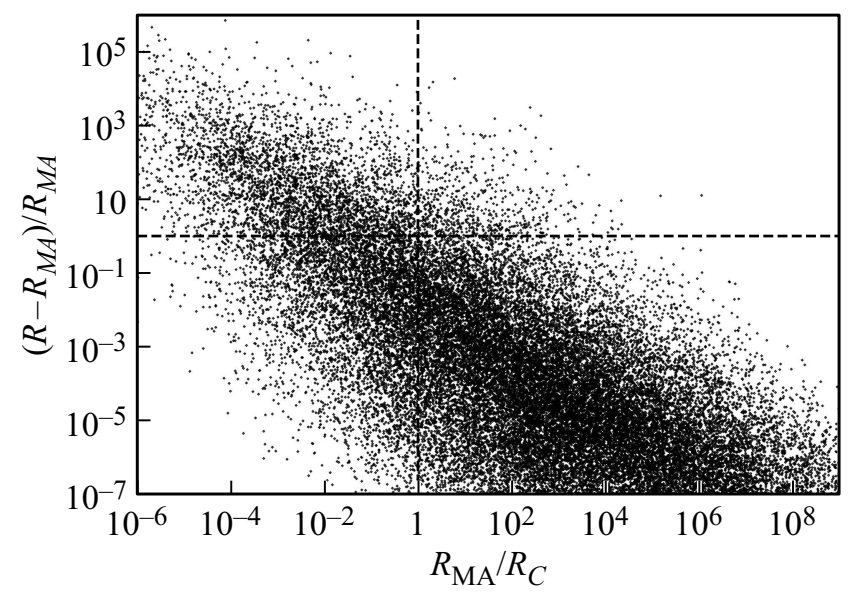

Рис. 5. Зависимость относительного изменения резистора за счет корреляции от значения сопротивления в модели Миллера-Абрахамса. Каждая точка на графике соответствует одному резистору.

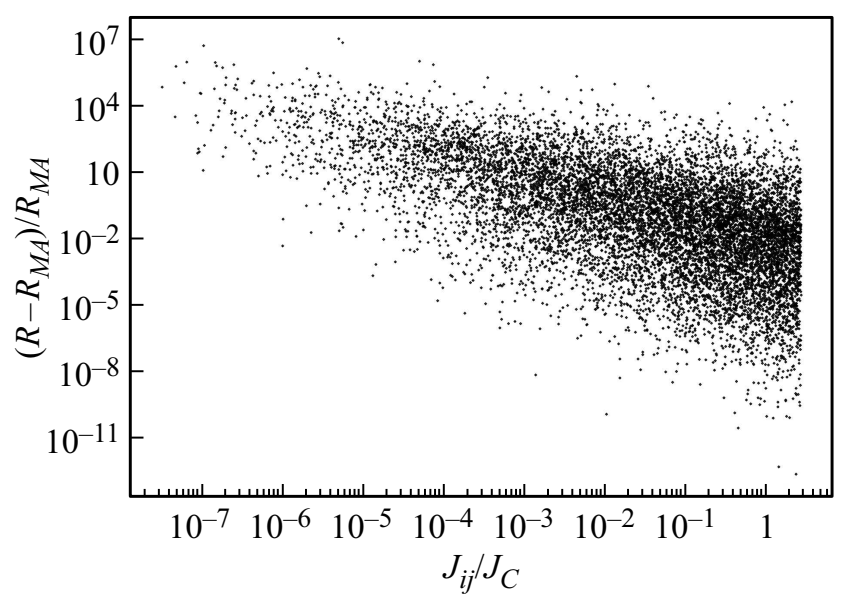

Рис. 6. Зависимость относительного изменения резистора за счет корреляции от протекающего через резистор тока. Каждая точка на графике соответствует одному резистору. 
плотности тока, текущего через макроскопическую систему. При этом в статистику включены только резисторы с сопротивлением меньше или порядка критического, потому что через остальные резисторы сильный ток заведомо течь не может. Корреляция между изменением сопротивления и протекающим током меньше, чем корреляция показанная на рис. 5. Тем не менее, сопротивление резисторов через которые течет большой ток, меняется в среднем слабее, чем резисторов, ток в которых мал. По-видимому, это связано с тем, что самые сильные изменения происходят в резисторах, оторваных от проводящего кластера, где электрон-дырочная пара, изображенная на рис. 3, $a$ не может уйти далеко от пары центров, на которой родилась.

\section{5. Заключение}

Мы показали, что система корреляционных кинетических уравнений может быть сведена к эквивалентной схеме с узлами, соответствующими прыжковым центрам. При сведении системы уравнений к такой схеме возникают нелокальные элементы (транссопротивления), связывающие напряжение на одном резисторе с током через другой резистор. Тем не менее, для систем в режиме закона Мотта достаточно точным является описание, не учитывающее таких нелокальных элементов. Это позволило нам исследовать вопрос о том, как меняются резисторы сетки сопротивлений из-за учета корреляции. Показано, что все они увеличиваются. Сильное увеличение наблюдается в первую очередь в резисторах, которые меньше критического сопротивления теории протекания.

\section{Конфликт интересов}

Авторы заявляют, что у них нет конфликта интересов.

\section{Список литературы}

[1] A. Miller, E. Abrahams. Phys. Rev. 120, 745 (1960).

[2] B.I. Shklovskii, A.L. Efros. Electronic Properties of Doped Semiconductors Springer, Berlin (1984).

[3] P.M. Richards. Phys. Rev. B 16, 1393 (1977)

[4] Е.И. Левин, В.Л. Нгуен, Б.И. Шкловский. ЖЭТФ 82, 1591 (1982); Sov. Phys. JETP 55, 921 (1982)

[5] O. Mermer, G. Veeraraghavan, T.L. Francis, Y. Sheng, D.T. Nguyen, M. Wohlgenannt, A. Kohler, M.K. Al-Suti, M.S. Khan. Phys. Rev. B 72, 205202 (2005).

[6] A.V. Shumilin, V.V. Kabanov. Phys. Rev. B 92, 014206 (2015).

[7] A.V. Shumilin, V.V. Kabanov, V.I. Dedieu. Phys. Rev. B 97, 094201 (2018).

[8] K.S. Chase, D.J. Thouless. Phys. Rev. B 39, 9809 (1989).

[9] O. Agam, I.L. Aleiner. Phys. Rev. B 89, 224204 (2014).

[10] A.V. Shumilin, Y.M. Beltukov. arXiv:1904.03103.

Редактор Е.Ю. Флегонтова 